\title{
Relação da prática de alimentação, higiene oral e fatores socioeconômicos com cárie precoce em escolares
}

\section{Relación de la práctica de alimentación, higiene oral y factores socioeconómicos con cárie precoce en escolares}

\section{Relationship of food practice, oral hygiene and socioeconomic factors with early dental caries disease in elementary school}

\author{
Liliane Santiago de Andrade \\ Ana Carolina Souza Torres ${ }^{1}$ \\ Naianny Rodrigues de Almeida \\ Maria Patrícia Rogério de Mendonça \\ Gilvan Lima Bezerra
}

RESUMO: Investigar a relação entre as práticas de alimentação, de higiene bucal, os fatores socioeconômicos e a prevalência de cárie precoce em pré-escolares, na idade de 3 a 6 anos, cobertos pela equipe da ESF no município de Maracanaú-CE. Trata-se de um estudo exploratório e transversal de abordagem quantitativa. Foi utilizada uma amostra por conveniência composta por 68 crianças na faixa etária de 3 a 6 anos. Exames clínicos odontológicos foram realizados para o diagnóstico de lesões de cárie. Questionários direcionados aos responsáveis das crianças permitiram a coleta de dados sobre as variáveis associadas à cárie, como práticas de alimentação, de higiene bucal e fatores socioeconômicos. Os dados foram analisados pelo teste do qui-quadrado ou extao de Fischer, seguido do modelo de regressão logística multinomial, sendo tabulados no Microsoft Excel e exportados para o software Statistical Packcage for the Social Sciences 17.0 de 2007, considerando uma confiança de 95\%. Das variáveis do estudo, Apresentaram significância para a CPI a criança ser portadora de alguma necessidade especial e a renda familiar $(\mathrm{p}<0,05)$, sendo as demais variáveis relacionadas a dieta, higienização bucal e fatores socioeconômicos não estatisticamente significativos $(p>0,05)$. Os resultados sugerem que a presença de alguma 
necessidade especial e uma baixa renda são fatores de risco para a CPI.

Palavras-chave: Cárie dentária, Pré-escolar, Saúde bucal, Prevalência, Fatores socioeconômicos.

ABSTRACT: To investigate the relationship between feeding practices, oral hygiene, socioeconomic factors and precocious caries prevalence in pre-school children aged 3 to 6 years, covered by the ESF team in the municipality of Maracanaú-CE. This is an exploratory and transversal study with a quantitative approach. A convenience sample of 68 children aged 3 to 6 years was used. Dental clinical examinations were performed for the diagnosis of caries lesions. Questionnaires addressed to the children responsible allowed the collection of data on the variables associated with caries, such as feeding practices, oral hygiene and socioeconomic factors. The data were analyzed by the chi-square or Fischer test, followed by the multinomial logistic regression model, tabulated in Microsoft Excel and exported to Statistical Packing for Social Sciences 17.0 of 2007, considering a confidence of $95 \%$. From the study variables, the child had a special need and family income $(\mathrm{p}<0.05)$. The other variables related to diet, oral hygiene and socioeconomic factors were not statistically significant $(\mathrm{p}<0.05)$. $>0.05)$. The results suggest that the presence of some special needs and a low income are risk factors for the ICC.

Key-words: Dental caries, Preschool, Oral health, Prevalence, Socioeconomic factors.

RESUMEN: La investigación de la relación entre las prácticas de alimentación, de higiene bucal, los factores socioeconómicos y la prevalencia de caries precoz en preescolares, a la edad de 3 a 6 años, cubiertos por el equipo de la ESF en el municipio de Maracanaú-CE. Se trata de un estudio exploratorio y transversal de abordaje cuantitativo. Se utilizó una muestra por conveniencia compuesta por 68 niños en el grupo de edad de 3 a 6 años. Se realizaron exámenes clínicos odontológicos para el diagnóstico de lesiones de caries. Los cuestionarios dirigidos a los responsables de los niños permitieron la recolección de datos sobre las variables asociadas a la caries, como prácticas de alimentación, de higiene bucal y factores socioeconómicos. Los datos fueron analizados por la prueba del chi-cuadrado o extracto de Fischer, seguido del modelo de regresión logística multinomial, siendo tabulados en Microsoft Excel y exportados al software Statistical Packcage for the Social Sciences 17.0 de 2007, considerando una confianza del 95\%. De las variables del estudio, presentaron significancia para la CPI el niño es portadora de alguna necesidad especial y la renta familiar $(\mathrm{p}<0,05)$, siendo las demás variables relacionadas a la dieta, higienización bucal y factores socioeconómicos no estadísticamente significativos ( $>>0), 05)$. Los resultados sugieren que la presencia de alguna necesidad especial y una baja renta son factores de riesgo para la CPI.

Palabras clave: Caries dentales, Pré-escolar, Salud bucal, Prevalência, Factores socioeconómicos.

\section{INTRODUÇÃO}

A cárie precoce da infância (CPI) é definida como uma lesão cariosa em dente decíduo em Tempus, actas de saúde colet, Brasília, 13(3), 139-152, set, 2019. Epub Jul/2020 ISSN 1982-8829 
crianças menores de 71 meses $^{1}$, sendo considerada um problema infantil comum no mundo ${ }^{2}$. Uma alimentação rica em sacarose associada a fatores como uma higiene bucal deficiente torna o sujeito mais suscetível ao desenvolvimento da doença ${ }^{3}$.

Apesar dos esforços empreendidos pela comunidade odontológica no controle da cárie, a CPI ainda preocupa pesquisadores, em especial nos países menos desenvolvidos ${ }^{4}$. O processo carioso, quando instalado, se não controlado, pode atingir estágios severos, repercutindo de maneira negativa na vida da criança, com consequências no seu desenvolvimento e crescimento. Representa um problema de saúde pública que atinge cerca de $27 \%$ das crianças de 18 a 36 meses de idade no Brasil, determinando dor, problemas na função da fala e mastigação, doenças sistêmicas e reflexos psicológicos ${ }^{1,5}$. As consequências da cárie severa na infância vão além dos efeitos primários (infecção e dor), afetando a saúde geral das crianças e podendo levar a desordens alimentares ${ }^{6}$.

As condições de saúde bucal podem ter um impacto negativo sobre o bem-estar funcional, social e psicológico das crianças e de suas famílias ${ }^{7}$. A criança de dois a seis anos de idade caminha no sentido da adapção mental e do equilíbrio de suas estruturas cognitivas. Estas últimas não lhe serão dadas diretamente pelo desenvolvimento biológico, mas resultarão da integração de um substrato nutricional orgânico e da busca de maneiras melhores de responder às solicitações do ambiente físico e social ${ }^{5}$.

A CPI interfere na qualidade de vida da criança e de sua família. O impacto dessa condição se faz sentir imediata e tardiamente, com diminuição na capacidade de aprendizagem pelas crianças, falta das mesmas à escola e absenteísmo dos pais no trabalho ${ }^{8,9}$. Essa situação patológica é capaz de refletir no comportamento social das crianças,que podem sofrer bullying pelos colegas. $\mathrm{O}$ tratamento da CPI, além de caro e muitas vezes invasivo (exodontia), é desgastante para a criança, sua família e equipe profissional ${ }^{8}$.

As circunstâncias da fase infantil, como status socioeconômicos e estrutura familiar, influenciam os atributos psicossociais, psicológicos e a qualidade de vida em relação à saúde oral nas crianças ${ }^{10}$. Pelo fato da cárie ser uma desordem de origem multifatorial, os fatores socioeconômicos, demográficos, cognitivos, hábitos de higiene bucal, dieta, acesso aos serviços odontológicos, renda familiar e grau de escolaridade devem ser considerados no seu estudo ${ }^{4,5}$.

A alta prevalência da cárie requer medidas interventivas adicionais no início da vida. É na infância que se encontram as condições para a aquisição de hábitos alimentares ${ }^{11}$. O desenvolvimento de programas relacionados à alimentação saudável torna-se relevante devido à influência das práticas alimentares no desenvolvimento da cárie dentária ${ }^{12}$.

O estudo sobre os fatores relacionados à cárie em pré-escolares, no cenário da Atenção Primária à Saúde Bucal, deve fornecer informações contextualizadas sobre os principais fatores envolvidos no desenvolvimento desse agravo. Diante disso, é importante o estudo sobre os fatores envolvidos 
na ocorrência dessa doença na época inicial da vida, uma vez que a experiência de cárie na dentição decídua é um precursor da doença na dentição permanente ${ }^{4}$.

O conhecimento sobre o impacto das condições de saúde bucal na qualidade de vida das crianças fornece medidas de resultado, contribuindo para uma melhor qualidade dos cuidados, além de proporcionar o desenvolvimento de políticas públicas com o objetivo de reduzir as desigualdades e de expandir o acesso a saúde, moradia, educação e trabalho para a população, contribuindo para uma boa qualidade de vida dos indivíduos ${ }^{13}$.

A realização de levantamentos estatísticos acerca dos procedimentos em saúde bucal mais realizados durante os atendimentos clínicos na Atenção Primária à Saúde, bem como investigações sobre as necessidades de tratamento odontológico de uma demanda populacional constituem trabalhos importantes. Por meio da análise estatística, podem-se investigar as principais demandas de saúde de uma população; com efeito, os levantamentos em saúde bucal constituem informação necessária para subsidiar o planejamento de ações de saúde, podendo orientar o desenvolvimento de políticas públicas ${ }^{14,15}$.

As investigações epidemiológicas são necessárias para informar sobre a prevalência das doenças bucais, estimar as necessidades de tratamento e determinar ações de promoção de saúde. Dessa forma, é possível um direcionamento dos recursos humanos e financeiros de acordo com as necessidades da população. É a partir dos dados coletados que se faz um planejamento adequado, de modo que seja possível executar e avaliar as ações de promoção da saúde, verificar a eficácia e produção dos serviços, além de permitir comparações em diferentes períodos de tempo ${ }^{14,15}$.

O Programa Saúde na Escola (PSE), instituído por meio do Decreto n 6.286 no ano de 2007 com o objetivo de promover ações de prevenção, promoção e atenção à saúde, engloba, como uma de suas ações, a avaliação de saúde e higiene bucal, cabendo às equipes de Saúde da Família (ESF) participar desse processo de interação entre a educação e a saúde ${ }^{16}$.

O estudo em ambiente escolar incentiva o desenvolvimento do tema saúde bucal no âmbito escolar, podendo envolver os familiares dos alunos por meio de ações de promoção e prevenção em saúde $^{17}$. É relevante o desenvolvimento de programas relacionados à prevenção e à educação em saúde na fase pré-escolar, sendo importante a participação dos pais nas atividades de educação em saúde desenvolvidas nas creches dos seus filhos ${ }^{3}$.

Durante a experiência de residente em Saúde da Família em uma Unidade Básica de Saúde (UBS) no município de Maracanaú, Ceará $(\mathrm{CE})$, em meio a um cenário próximo do que anteriormente exposto, verificou-se a baixa demanda de pré-escolares na UBS visando cuidados da saúde oral, donde a necessidade de realizar a busca ativa e de promover uma maior atenção a esse público.

O presente estudo tem o objetivo de investigar a relação entre as práticas de alimentação, de higiene bucal, os fatores socioeconômicos e a prevalência de cárie precoce em pré-escolares, na Tempus, actas de saúde colet, Brasília, 13(3), 139-152, set, 2019. Epub Jul/2020 ISSN 1982-8829 
idade de 3 a 6 anos, cobertos pela equipe da ESF no município de Maracanaú-CE.

\section{METODOLOGIA}

Tratou-se de um estudo exploratório e transversal de abordagem quantitativa. A pesquisa teve como cenário uma creche-escola pertencente à área de abrangência da ESF da qual a cirurgiãdentista fez parte enquanto residente, de uma UBS no município de Maracanaú-CE, no período de janeiro a fevereiro do ano de 2017.

A coleta de dados foi realizada pela profissional cirurgiã-dentista devidamente capacitada e residente pelo programa de Residência Multiprofissional em Saúde da Família e Comunidade, promovido pela Escola de Saúde Pública do Ceará

A primeira etapa da coleta constou de um exame clínico odontológico em ambiente escolar com auxílio de espátulas de madeira, sob luz natural, nas crianças participantes da pesquisa, verificando a presença de cárie dentária, definida para a odontologia tradicional como cavidade no esmalte e/ou dentina, considerando livres de cárie os indivíduos que não apresentam essas lesões ${ }^{17}$. $\mathrm{Na}$ segunda etapa, aplicou-se um instrumento do tipo questionário semiestruturado, composto de 16 questões, direcionado aos pais ou responsáveis pelas crianças, com assuntos relacionados a dieta, higiene bucal dos pré-escolares e fatores socioeconômicos da família. O questionário e o Termo de Consentimento Livre e Esclarecido (TCLE) foram entregues aos responsáveis por meio de anexo à agenda escolar do aluno ou pessoalmente pelos professores no momento em que os responsáveis chegavam à creche-escola para deixar ou buscar as crianças.

No presente estudo, foi utilizada uma amostra de conveniência, uma vez que a pesquisadora não recebeu da instituição escolar a listagem com os nomes dos alunos matriculados, e teve dificuldades em acessar as crianças nos espaços escolares. A população de escolha constituiu de crianças na faixa etária de três a seis anos por estarem na fase de dentição decídua, pela baixa frequência desse público na consulta odontológica na UBS e por ser uma população de mais fácil acesso em ambiente escolar, onde a pesquisa foi realizada.

Para definição da amostra, a partir de uma população de 84 crianças, foi utilizado o cálculo para populações finitas através do programa software Statistical Packcage for the Social Sciences 17.0 de 2007 , tomando como base um erro amostral de 5\%, nível de confiança de 95\%, poder do teste de $80 \%$ e prevalência de violência mínima do desfecho (PRESENÇA DE CÁRIE) de $66 \%$, baseado na frequência de crianças com cárie dentária estimada pelo SBBrasil 2010 de 66\%. Dessa forma, a amostra calculada foi de 68 CRIANÇAS.

A pesquisa teve como critérios de inclusão crianças na faixa etária de três a seis anos, de ambos os sexos, matriculadas em uma creche de cobertura da equipe da ESF de uma UBS no município de 
Maracanaú-CE, sendo excluídas as crianças que não colaboraram no momento no exame clínico.

Os dados foram tabulados no Microsoft Excel e exportados para o software Statistical Packcage for the Social Sciences (SPSS) 17.0, no qual as análises estatísticas foram realizadas adotando um nível de confiança de $95 \%$.

A estatística descritiva contou com a expressão da frequência absoluta e relativa das variáveis desfecho (cárie dentária) e independentes (sexo da criança, idade do responsável, parentesco do responsável, renda mensal familiar, saneamento básico, escolaridade do responsável, filho com necessidades especiais, ida do filho ao dentista, frequência de escovação, uso de pasta de dente com flúor, frequência de uso do açúcar em alimento pastoso, frequência de uso do açúcar no líquido, tipo de água que ingere, frequência da ingestão de alimentos doces, ingestão de mamadeiras com açúcar, ingestão de mamadeira com açúcar quando dorme, aleitamento materno, leva lanche para escola, número de pessoas na residência), que posteriormente foram confrontadas por meio do teste do qui-quadrado ou exato de Fisher, com a finalidade de investigar os fatores de risco à cárie na infância. Adicionalmente, os dados foram agrupados e submetidos ao modelo de regressão logística multinomial.

Este estudo foi conduzido ao Comitê de Ética em Pesquisa da Escola de Saúde Pública do Ceará e aprovado sob o parecer favorável de número 1.880.235. Os participantes da pesquisa assinaram um Termo de Consentimento Livre e Esclarecido (TCLE) que explicou a realização do estudo, os objetivos, riscos e benefícios aos quais estariam expostos, de acordo com as Diretrizes e Normas Regulamentadoras do Conselho Nacional de Saúde (Resolução nº 466/2012).

A pesquisa não apresentou nenhum risco direto à saúde física, visto que tratou-se apenas de aplicação de questionários e exame bucal a olho nu para verificar a presença de cárie dentária. Os riscos foram mínimos e se referiram à possibilidade de constrangimento e/ou desconforto com algum questionamento.

\section{RESULTADOS}

A amostra era composta por 73,5\% (50) de meninas e 26,5\% (18) de meninos. Das crianças examinadas, 58,8\% (40) eram livres de cárie e 41,2\% (28) tinham a doença (Tabela 1).A maioria das famílias apresentou uma baixa renda mensal, com 55,9\% (38) possuindo uma renda de até um salário mínimo, que corresponde a novecentos e trinta e sete reais no ano do estudo, e 32,4\% (22) apresentam entre um e dois salários mínimos. A maioria dos responsáveis pelas crianças possuía o ensino médio completo $39,7 \%$ (27) e uma minoria 1,5\% (1) havia concluído o ensino superior. A maioria das crianças 57,4\% (39) do estudo não tinha frequentado um dentista (Tabela 1).

Tabela 1: Dados relacionados às práticas de alimentação, higiene bucal e fatores socioeconômicos de crianças e seus responsáveis no município de Maracanaú, CE (2017). 
A associação entre a CPI, a prática de alimentação infantil, os fatores socioeconômicos e a frequência de higiene bucal, por meio do teste do qui-quadrado ou exato de Fisher, é evidenciada na Tabela 2. Observa-se que a variável "presença de necessidade especial" apresentou significância estatística $(\mathrm{p}=0,025)$ quando confrontada à variável "presença de CPI". Dos pré-escolares livres de cárie $(n=40)$, nenhum era portador de necessidade especial, e dos que tinham cárie $(n=28)$, $14,3 \%$ apresentavam alguma necessidade especial.

Tabela 2: Associação entre a cárie precoce da infância e variáveis relacionadas no Município de Maracanaú, CE (2017).

Em relação ao aleitamento materno, a maior prevalência foi de crianças que mamaram até seis meses de idade, compondo 29,4\% (20) do total (Tabela 1). Na Tabela 2, nota-se que, das crianças livres de cárie, mais da metade 75\% (30) receberam aleitamento materno, e das que tinham lesão cariosa, 28,6\%(8) não mamaram.

Com os dados coletados nesse estudo e seguindo o modelo de regressão logística multinomial, observou-se que a renda familiar mensal acima de um salário mínimo reduz 0,18 vezes a frequência de CPI, e a presença de alguma necessidade especial aumenta 4,4 vezes essa frequência. As demais variáveis não apresentaram associação significativa com a cárie dental (Tabela 3).

Tabela 3: Modelo multinomial da cárie precoce da infância em relação à dieta, higienização bucal e aos fatores socioeconômicos, Maracanaú/CE, 2017.

\section{DISCUSSÃO}

$\mathrm{Na}$ presente pesquisa observou-se que mais da metade $(58,8 \%)$ das crianças eram livres de cárie, em concordância com outros estudos, como o de Alcântara et $\mathrm{al}^{3}$, que verificou fatores associados à cárie em pré-escolares de 4 meses a 6 anos de idade em uma creche no município de Piracicaba-SP, onde $81,7 \%$ dos pré-escolares eram livres de cárie. No estudo de Almeida et al ${ }^{18}$, que determinou a prevalência e gravidade da cárie dental em pré-escolares de cinco anos de idade, $50,4 \%$ das crianças estavam livres da doença. No estudo de Feldens et al ${ }^{12}$, que avaliou crianças de 4 anos de idade, 37\% não tinham sinais clínicos de cárie.

Parisotto et $a{ }^{4}{ }^{4}$ verificaram a prática de alimentação, higiene bucal e fatores socioeconômicos na prevalência da cárie precoce da infância em pré-escolares de 3 a 4 anos de idade que frequentavam pré-escolas públicas no município de Itatiba-SP, observando que a frequência diária do uso de mamadeira contendo líquido açucarado, a frequência diária de açúcar na forma líquida e total, e o fato da criança ser colocada para dormir com a mesma contendo conteúdo açucarado não apresentaram associação significativa com a CPI. No estudo em questão, também não foi observada uma relação de significância entre a frequência diária do consumo de açúcar na forma líquida e pastosa, a frequência diária do uso da mamadeira contendo conteúdo açucarado e do uso da mesma durante o sono da criança com a cárie em pré-escolares. Mesmo diante desses 
resultados, ressalta-se que o uso de mamadeira com conteúdo açucarado e uma higienização oral deficiente após as mamadas podem acarretar o surgimento de cárie ${ }^{19,20}$.

No estudo realizado por Alcântara et al $^{3}$, que avaliou a condição de saúde bucal de pré-escolares de uma creche e os fatores associados à ocorrência de cárie, a maioria $(83,5 \%)$ das crianças fazia uso de mamadeira noturna, assim como no presente estudo, no qual todas as crianças usaram mamadeira contendo conteúdo açucarado durante o sono, com uma frequência de pelo menos uma vez durante esse período. No entanto, em ambos os estudos, esse dado não foi de relevância para a CPI.

Folayan et al ${ }^{1}$ determinaram os indicadores de risco e prevalência da CPI em uma população suburbana na Nigéria, onde constataram como indicadores de risco para a cárie dental o sexo da criança, o conhecimento das mães a respeito de saúde oral, o consumo de lanches contendo açúcar entre as refeições, em uma frequência de três ou mais vezes ao dia, e o estado de higienização bucal. Nesse mesmo estudo, a idade das crianças também foi significativamente associada à CPI, no qual a proporção de crianças com cárie aumentou com o aumento da idade. Outros fatores como o status socioeconômico, o número de membros da família, a frequência de escovação, o uso de pasta de dente com flúor, a frequência da criança ao dentista, o estado de higiene bucal e práticas de alimentação infantil (formas de aleitamento materno, amamentação noturna, duração da amamentação) não foram de significância para a CPI. A literatura mostra que o uso de pasta de dente fluoretada apresenta efeito inibidor da cárie na dentição permanente, com poucas evidências na dentição decídua ${ }^{21}$. No presente estudo, de forma semelhante ao mencionado anteriormente, somente os fatores renda e necessidade especial foram significativos para a CPI, sendo os demais, biológicos e não biológicos, em concordância com o estudo de Folayan et al ${ }^{1}$, não relevantes.

Segundo Queiroz et al ${ }^{17}$, o maior risco do desenvolvimento de doenças bucais,como a cárie dentária e a doença periodontal em pacientes portadores de alguma necessidade especial sofre influência de fatores como limitações físicas e mental, uma dieta rica em carboidratos, dificuldade e negligência da higiene bucal desses pacientes por parte dos seus responsáveis, o que favorece o acúmulo de placa bacteriana e o desenvolvimento das patologias bucais.

Alguns estudos ${ }^{3,18}$ identificaram o sexo como um fator de risco para a CPI. Almeida et al ${ }^{18}$ observaram que as crianças do sexo feminino, na idade de cinco anos, estavam entre as que mais apresentavam necessidade de tratamento para a cárie dentária. No presente estudo, a maioria $(67,9 \%)$ das crianças com cárie era do sexo feminino. Deve ser levado em consideração que a maioria da amostra do estudo era composta por crianças do sexo feminino, correspondendo a $73,5 \%$ (50) do total. No estudo de Kremer et $\mathrm{al}^{22}$, foi observado que as crianças do sexo feminino apresentaram uma maior possibilidade de terem realizado algum atendimento odontológico. A irrupção dos dentes ocorre com mais frequência primeiro no sexo feminino; a depender da idade da criança e da experiência de cárie, diferenças em relação ao gênero em função do risco relacionado ao tempo de exposição dos elementos dentários podem ser encontradas ${ }^{23}$. 
A presente pesquisa corroborou com os achados de Ortiz et $\mathrm{al}^{24}$, nos quais a maioria dos pais, responsáveis pelas crianças participantes do estudo, apresenta uma baixa renda familiar. Somente um quarto das crianças $(25,05 \%)$ frequentaram um dentista e a experiência de cárie foi notada na minoria $(16,4 \%)$. No presente estudo, a maior parte das famílias possuía uma baixa renda mensal, com 55,9\% possuindo uma renda de até um salário mínimo, e 32,4\% de um a dois salários mínimos. A maioria dos pré-escolares (39\%) não havia frequentado um dentista e a experiência de cárie foi observada em somente $28 \%$ das crianças. Relacionando a atividade de cárie com o nível econômico, observou-se que, das crianças com cárie, em quase metade (46,4\%), a família possuía uma renda mensal de no máximo um salário mínimo.

No estudo de Alcântara et $\mathrm{al}^{3}$, os fatores associados à cárie dental foram sexo, renda e escolaridade dos pais ou responsáveis. A renda familiar mais prevalente (43\%) estava entre $\mathrm{R} \$ 500,00$ e $\mathrm{R} \$ 1.000,00$, valor entre um a dois salários mínimos no ano do estudo, e as crianças, cujo arranjo familiar apresentava a menor renda, tinham uma maior predominância de cárie. Corroborando com os resultados desse estudo, na presente pesquisa a maior renda familiar encontrada estava entre um a dois salários mínimos e, em $85,7 \%$ das crianças com cárie, a família apresentava uma renda de no máximo dois salários mínimos.

Assim como no estudo de Gomes et $\mathrm{al}^{7}$, que concluiu que as lesões cariosas cavitadas estabeleciam uma associação significativa com a renda familiar, em outros estudos ${ }^{3,25}$, foi observada essa relação do nível econômico com a cárie dentária, onde a doença era mais frequente em crianças de mais baixo nível econômico. A presente pesquisa mostrou que uma renda familiar mensal acima de um salário mínimo reduz 0,18 vezes a frequência de CPI.

Scarpelli et a ${ }^{13}$ avaliaram o impacto das condições de saúde bucal na qualidade de vida em uma população de pré-escolares brasileiros, identificando a influência do perfil sociodemográfico e as percepções quanto à saúde geral e ao estado de saúde oral na qualidade de vida do indivíduo. Os resultados mostraram um impacto negativo na qualidade de vida relacionada à saúde bucal associada à experiência de cárie e renda familiar mensal.

Há na literatura estudos que relatam que o uso dos serviços odontológicos é proporcional ao nível econômico, onde as famílias com baixo nível econômico têm mais dificuldades de acesso aos serviços de saúde bucal ${ }^{26}$. No presente estudo, as famílias de baixo nível econômico não tinham condições de ter acesso aos serviços odontológicos no âmbito particular, dependendo somente do que o serviço público poderia oferecer, o que, devido às limitações e dificuldades na saúde pública, não era o suficiente para atender a todas as demandas de saúde bucal dos indivíduos. Segundo Santhosh et $\mathrm{al}^{27}$, a saúde bucal de crianças está relacionada às dimensões sociais, incluindo educação e renda dos pais. Os pais que tinham baixo nível de escolaridade tinham pouco conhecimento da importância da saúde bucal na primeira infância, relataram achar que não precisavam se preocupar com a saúde bucal das crianças na fase de dentição decídua, uma vez que era uma dentição que seria "trocada" posteriormente, além de terem dificuldade de perceberem os primeiros sinais clínicos da 
cárie.

Alcântara et $\mathrm{al}^{3}$ evidenciaram que, na maioria das crianças com cárie, os pais apresentavam uma baixa escolaridade. Contudo, pode-se observar a importância da educação dos responsáveis das crianças, como já mencionado em outros estudos ${ }^{28}$, cujos autores relataram a importância do papel das mães na educação dos seus filhos, principalmente no processo de aquisição de bons hábitos de higiene bucal.

Alcântara et $\mathrm{al}^{3}$ constataram que $36,4 \%$ dos pais ou responsáveis pelas crianças tinham estudado até o ensino fundamental e a maioria $(63,6 \%)$, além do ensino fundamental. No presente estudo, a maioria $(39,7 \%)$ tinha o ensino médio completo e somente $1,5 \%$ havia concluído o ensino superior. Associando com a cárie dentária, nota-se que, em mais da metade $(57,1 \%)$ das crianças com cárie, o familiar responsável não havia concluído os estudos, possuindo, no máximo, o ensino médio incompleto.

Outros estudos ${ }^{29}$ mostram a relação entre a baixa escolaridade dos pais com a CPI como uma falta de conhecimento dos responsáveis sobre saúde bucal, não possuindo saberes sobre hábitos de uma saúde oral saudável, de modo a contribuir para que ofereçam alimentos cariogênicos às crianças.

É importante que pais e demais responsáveis pelas crianças tenham acesso às orientações de prevenção contra a cárie na primeira infância, incluindo informações quanto ao período da primeira visita ao dentista da criança, devendo ser priorizadas aquelas com mais de um filho e de baixa renda ${ }^{30}$.

O desenvolvimento de medidas interventivas com o objetivo de melhorar a conscientização sobre os cuidados em saúde bucal por parte dos pais e demais familiares é estratégia relevante para se promover saúde bucal na infância. A desinformação das famílias envolvidas no cuidado em saúde bucal das crianças apresenta associação com um maior número de cáries ${ }^{2}$ na primeira infância.

\section{CONCLUSÃO}

De acordo com os dados encontrados nessa pesquisa, dentre os aspectos biológicos e não biológicos relacionados às práticas de alimentação, higiene bucal e fatores socioeconômicos, concluiu-se que os fatores renda familiar e necessidade especial foram os indicadores de relevância para a cárie precoce da infância.

Constatou-se, no presente estudo, que a criança ser portadora de alguma necessidade especial aumenta 4,4 vezes a frequência de CPI e uma renda mensal familiar acima de um salário mínimo reduz 0,18 vezes essa frequência, sendo os demais fatores não significativos para a CPI. 
Ressaltamos a importância do desenvolvimento de políticas públicas para o estabelecimento de programas curativos, preventivos e de promoção em saúde bucal específicos para pacientes com necessidade especiais.

Dentre as limitações do estudo, encontram-se o fato de que a idade da criança não foi investigada, utilizando-se apenas uma faixa etária, a dificuldade de realização do exame bucal em algumas crianças e o preenchimento e retorno do questionário por parte dos responsáveis pelos pré-escolares.

Em decorrência da assistência clínica gerada ao longo dos anos, a odontologia apresenta uma grande demanda reprimida, sendo importante o trabalho da equipe da Estratégia Saúde da Família capilarizando o acesso aos cuidados em saúde. Com a criação da Política Nacional de Saúde Bucal e a inserção do cirurgião-dentista na equipe ESF, foi possível o acesso aos cuidados em saúde bucal por populações com vulnerabilidades sociais.

Esse estudo sugere a realização de futuras pesquisas com o intuito de aprofundar os apontamentos evidenciados e suprir as possíveis lacunas.

\section{REFERÊNCIAS}

1. Folayan MO, Kolawole KA, Oziegbe EO, Oyedele T, Oshomoji OV, Chukwumah NM, et al. Prevalence, and early childhood caries risk indicators in preschool children in suburban Nigeria. BMC Oral Health 2015 [acesso 2017 dez 10]; 15:72. doi: 10.1186/s12903-015-0058-y. Disponível em: https://bmcoralhealth.biomedcentral.com/track/pdf/10.1186/s12903-015-0058$\mathrm{y}$ ? site $=$ bmcoralhealth.biomedcentral.com

2. Kramer PF, Feldens CA, Ferreira SH, Brevian J, Rodrigues PH, Peres MA. Exploring the impact of oral diseases and disorders on quality of life of preschool children. Community Dent Oral Epidemiol 2013; 41(4): 327-335.

3. Alcântara TH, Batista MJ, Gibilini C, Ferreira NP, Sousa MLR. Fatores associados à saúde bucal de pré-escolares inseridos em programa educativo preventivo no município de Piracicaba/ SP. RPG Rev Pós Grad. 2011; 18(2): 102-107.

4. Parisotto TM, Oliveira CS, Silva CMS, Almeida MEC, Rodrigues LKA, Santos MN. A importância da prática de alimentação, higiene bucal e fatores sócio-econômicos na prevalência da cárie precoce da infância em pré-escolares de Itatiba-SP. Ver Odontol Bras Centra. 2010; 19(51): 333-339.

5. Feitosa S, Colares V. As repercussões da cárie precoce na infância na qualidade de vida de pré-escolares. Rev Ibero-am Odontopediatria Odontologia Bebê. 2003; 6(34): 542-548. 
6. Moreira RS, Nico LS, Tomita NE, Ruiz T. A saúde bucal do idoso brasileiro: revisão sistemática sobre o quadro epidemiológico e acesso aos serviços de saúde bucal. Cad Saúde Pública. 2005; 21(6): 1665-1675. doi: 10.1590/S0102-311X2005000600013

7. Gomes MC, Pinto-Sarmento TCA, Costa EMMB, Martins CC, Granville-Garcia AF, Paiva SM. Impact of oral health conditions on the quality of life of preschool children and their families: a cross-sectional study. Health Qual Life Out comes 2014 Apr 18; 12(55): 1-5. doi: 10.1186/1477-7525-12-55.

8. Losso EM, Tavares MCR, Silva JYB, Urban CA. Cárie precoce e severa na infância: uma abordagem integral. Jornal de Pediatria 2009; 85(4): 295-300. doi: 10.1590/S002175572009000400005 .

9. Ribeiro GL, Gomes MC, de Lima KC, Martins CC, Paiva SM, Granville-Garcia AF. Work absenteeism by parents be cause of oral conditions in preschoolchildren. Int Dent J. 2015 Dec; 65(6): 331-337. doi: 10.1111/idj.12184.

10. Kumar S, Kroon J, Lalloo R. A systematic review of the impact of parental socio-economic status and home environment characteristics on children's oral health related quality of life. Health Qual Life Outcomes. 2014; 12(41): 1-15. doi: 10.1186/1477-7525-12-41.

11. Molina MC, Lopéz PM, Faria CP, Cade NV, Zambona E. Preditores socioeconômicos da qualidade da alimentação de crianças. Saúde Publica. 2010 [acesso 2017 dez 10]; 44(5): 785 792. doi:10.1590/S0034-89102010005000036. Disponível em: http://www.scielo.br/scielo. php?script=sci_arttext\&pid=S0034-89102010000500003

12. Feldens CA, Giugliani ERJ, Duncan BB, Drachler ML, Vítolo MR. Long-term effectiveness of a nutritional program in reducing early childhood caries: a randomized trial. Community Dent Oral Epidemiol. 2010 Aug; 38(4): 324-332. doi: 10.1111/j.16000528.2010.00540.x.

13. Scarpelli AC, Paiva SM, Viegas CM, Carvalho AC, Ferreira FM, Pordeus IA. Oral healthrelated quality of life among Brazilian preschool children. Community Dent Oral Epidemiol. 2013 Aug; 41(4): 336-344. doi: 10.1111/cdoe.12022.

14. Domingos, PAS, Ribeiro D, Dinelli W, Staufackar CA, Campo JADB. Aspectos epidemiológicos da saúde bucal de crianças em um município brasileiro. Jornal de Pediatria. 2010; 46(2): 82-87.

15. Oliveira AGRC, Unfer B, Costa ICC, Guimarães LOC, Saliba NA. Levantamentos epidemiológicos em saúde bucal: análise da metodologia proposta pela Organização Mundial da Saúde. Rev; Bras Epidemiol. 1998; 1(2):177-189. 
16. Brasil. Ministério da Saúde. Secretaria de Atenção à Saúde. Departamento de Atenção Básica. Saúde na escola. Brasília: Ministério da Saúde, 2009.

17. Pauleto AR, Pereira ML, Cyrino EG. Saúde bucal: uma revisão crítica sobre programações educativas para escolares. Ciênc Saúde Coletiva. 2004; 9(1): 121-130. doi: 10.1590/S141381232004000100012.

18. Almeida TF, Cangusso MC, Chaves SC, Castro e Silva DI, Santos SC. Condições de saúde bucal de crianças na faixa etária pré-escolar, residentes em áreas de abrangência do Programa Saúde da Família em Salvador, Bahia, Brasil. Rev Bras Saude Mater Infant. 2009; 9(3): 247-252. doi: 10.1590/S1519-38292009000300003.

19. Ditterich RG, Romanelli MV, Rastelli MC, Wambier DS. Cárie de acometimento precoce: uma revisão. Cienc Biol Saúde. 2004;10(3):33-41.

20. Rezende LN, Santos FCS, Neto MS, Santos, FS. Cárie rampante de mamadeira em crianças de 2 a 5 anos: revisão de literatura. J Manag Prim Health Care. 2014; 5(2): 219-229.

21. Marinho VCC, Higgins JPT, Logan S, Sheiham A. Fluoride tooth pastes for preventing dental caries in children and adolescentes. Cochrane Database Syst Rev. 2003 Jan 20; 1(1): 1-12. doi: 10.1002/14651858.CD002278.

22. Kramer PF, Ardenghi TM, Ferreira S, Fischer LA, Cardoso L, Feldens CA. Utilização de serviços odontológicos por crianças de 0 a 5 anos de idade no Município de Canela, Rio Grande do Sul, Brasil. Cad. Saúde Pública 2008 Jan;24(1):150-156. doi: 10.1590/S0102311 X2008000100015

1. 23. Cypriano S, Hugo FN, Sciamarelli MC, Tôrres LHN, Sousa MLR, Wada RS.Fatores associados à experiência de cárie em escolares de um município com baixa prevalência de cárie dentária. Ciênc. Saúde Coletiva 2011 Oct; 16(10): 4095-4106. doi: 10.1590/S141381232011001100015 .

24. Ortiz FR, Tomazoni F, Oliveira MDM, Piovesan C, Mendes F, Ardenghi TM. Toothache, Associated Factors, and Its Impact on Oral Health-Related Quality of Life (OHRQoL) in Preschool Children. Brazilian Dental Journal. 2014; 25(6): 546-553. doi: 10.1590/01036440201302439

25. Carvalho DM, Salazar M, De Oliveira BH, Coutinho ESF. O uso de vernizes fluoretados e a redução da incidência de cárie dentária em pré-escolares: uma revisão sistemática. Rev Bras Epidemiol. 2010; 13(1): 139-149. doi: 10.1590/S1415-790X2010000100013.

26. Araújo CS, Lima RC, Peres MA, Barros AJD. Utilização de serviços odontológicos e 
fatores associados: um estudo de base populacional no Sul do Brasil. Cad. Saúde Pública. 2009; 25(5): 1063-1072. doi: 10.1590/S0102-311X2009000500013.

27. Santhosh KJT, Prabu D, Suhas K. Socio-behavioral variables effecting oral hygiene and periodontal status of 12 year-old school children of Udaipur district. Odontostomatol Trop. 2013 Mar; 36(141): 27-33.

28. Fadel CB, Saliba NA. Aspectos sócio-dentais e de representação social da cárie dentária no contexto materno infantil. RGO. 2009; 57(3): 303-309.

29. Wyne AH, Adenubi JO, Shalan T, Khan N. Alimentary and socioeconomic characteristics of children that developed caries in a Saudian population. Pediatr Dent. 1995; 17(7): 451-454.

30. Azevedo MS, Romano AR, Santos IS, Censi MS. Knowledge and beliefs concerning early childhood caries from mothers of children ages zero to 12 months. Pediatr Dent. 2014; 36(3): 9599.

Artigo apresentado em maio de 2018 Artigo aprovado em julho de 2019 Artigo publicado em julho de 2020 